\title{
Synchronous detection technique for temporal fringe pattern analysis
}

\author{
J. Villa, J.A. Gómez-Pedrero*, J.A. Quiroga \\ Departamento de Óptica, Facultad de Ciencias Fisicas, Universidad Complutense de Madrid, \\ Ciudad Universitaria s/n, Madrid 28040, Spain
}

Received 25 October 2001; received in revised form 19 December 2001; accepted 14 January 2002

\begin{abstract}
An alternative method for temporal evaluation of fringe patterns is proposed. The method is based on the application of a synchronous detection system for processing the temporal irradiance fluctuations generated when the sensitivity of the optical set-up is changed. The technique requires an easy computing implementation and presents good noise rejection characteristics. In particular, the proposed method does not require to store the whole set of fringe images as it happens with Fourier techniques which allows for a faster data processing. Results of this method proved on synthetic fringe images as well as in real experiments are presented. (c) 2002 Published by Elsevier Science B.V.
\end{abstract}

\section{Introduction}

Optical techniques commonly require the analysis of fringe patterns to measure a wide quantity of physical parameters such as surface profile, displacement and refractive index. Several techniques for analyzing fringe patterns have been developed [1]. In recent years [2-7], several techniques has been developed for the temporal evaluation of phase maps. These temporal techniques have important advantages compared with spatial ones since the phase at each point is recovered independently, allowing the evaluation of discontinuous phase functions. An additional advantage derived from the independent evaluation of the

\footnotetext{
${ }^{*}$ Corresponding author. Fax: +34-1-394-46-74.

E-mail addresses: jvilla@fis.ucm.es (J. Villa), jagomezp@fis.ucm.es (J.A. Gómez-Pedrero).
}

phase at each image point is that errors generated in noisy regions do not propagate over the full field.

The most important temporal techniques for fringe pattern analysis are the methods developed by Huntley and Saldner [2,3], and the temporal Fourier techniques [4-6]. Huntley's method is based on the application of a phase-stepping algorithm when the sensitivity of the optical arrangement is changed over the time. In this way, it is obtained, for each image point, a set of wrapped phase values with different sensitivities. After performing a 1-D unwrapping operation along the time axis, we get the final unwrapped phase for each image point. The phase unwrapping in this method is implicit, therefore no further spatial unwrapping must be applied. This method was applied to surface profiling using a Michelson interferometer by Saldner and Huntley [7]. As in Huntley's method, in the temporal Fourier 
techniques the sensitivity of the optical set-up is changed along the time to get a temporal sinusoidal signal generated by the irradiance fluctuations at each pixel. From these temporal signals, the phase is obtained for each point by applying the Fourier transform technique. The temporal Fourier analysis applied to surface profiling was reported by Takeda and Yamamoto [4], and Joenathan et al. [5]. An application to deflectometry for the measurement of power in ophthalmic lenses with a limited number of images was proposed by Quiroga and Gómez-Pedrero [6].

In this work we propose an alternative approach for temporal fringe pattern analysis. This technique is applied to the temporal irradiance fluctuations generated by a linear variation of the sensitivity. The method is based on the temporal synchronous detection of the phase at each image point. Main advantages of the technique are as follows: (1) It is easy to implement and no complete storage of the images is required because it is based on the convolution operation rather than Fourier transformation. (2) It presents good noise rejection characteristics due to the application of a convolution filter. (3) The presented technique is not so sensitive to the conditions of symmetry of the temporal signal as happens with the Fourier technique. (4) Finally, the proposed technique, does not require a big quantity of images as the Huntley's method, which requires four times the number of samples due to the phase-stepping algorithm.

This paper is organized as follows. A theoretical description of the proposed technique is presented in Section 2. The results of numerical experiments carried out to analyze the performance of the technique are presented in Section 3. Experimental results of the application to surface profiling are presented in Section 4. Finally, conclusions are given in Section 5.

\section{Synchronous detection for temporal phase-mea- surement}

\subsection{Temporal phase-measurement}

Considering that a fringe pattern image is represented by $g(x, y)=a(x, y)+b(x, y) \cos [\Phi(x, y)+\Omega(x, y)]$,

where $a(x, y)$ and $b(x, y)$ represent the background illumination and the amplitude modulation, respectively. The field $\Phi(x, y)$ represents the phase to be detected which is related with the physical quantity to be measured, and $\Omega(x, y)$ represents the carrier term. Defining $f(x, y)$ as the physical quantity to be measured and $\varepsilon(t)$ the sensitivity of the optical system that depends on time, Eq. (1) can be rewritten as

$$
\begin{aligned}
g(x, y ; t)= & a(x, y ; t)+b(x, y ; t) \\
& \times \cos (\varepsilon(t) \cdot[f(x, y)+k(x, y)]),
\end{aligned}
$$

where we suppose that

$\Phi(x, y ; t)=\varepsilon(t) \cdot f(x, y)$ and

$\Omega(x, y ; t)=\varepsilon(t) \cdot k(x, y)$.

Eq. (2) represents a set of fringe images for different sensitivities, where $t=1,2,3 \ldots, N$.

If we impose that the sensitivity varies linearly, that is, $\varepsilon(t)=\varepsilon_{0}+\mu \cdot(t-1)$, we can observe from Eq. (2) that, for every image point, we shall have a temporal cosine function with frequency proportional to $f(x, y)+k(x, y)$. Fig. 1 shows a schematic diagram of the generation of the irradiance fluctuations over time at different image locations. Let

$$
\begin{aligned}
\Theta(x, y ; t) & =\varepsilon(t) \cdot[f(x, y)+k(x, y)] \\
& =\alpha(x, y)+\beta(x, y) \cdot(t-1),
\end{aligned}
$$

where

$$
\begin{aligned}
& \alpha(x, y)=\varepsilon_{0} \cdot[f(x, y)+k(x, y)], \\
& \beta(x, y)=\mu \cdot[f(x, y)+k(x, y)] .
\end{aligned}
$$

Then

$$
\begin{aligned}
g(t) & =a(t)+b(t) \cos [\Theta(t)] \\
& =a(t)+b(t) \cos [\alpha+\beta \cdot(t-1)], \\
t & =1,2,3, \ldots, N .
\end{aligned}
$$

Eq. (6) represents a function of $x$ and $y$ but the spatial dependence has been removed for simplicity. As it can be seen, to recover $f(x, y)$ we just have to calculate the value of the temporal frequency $\beta$ at every image point. 


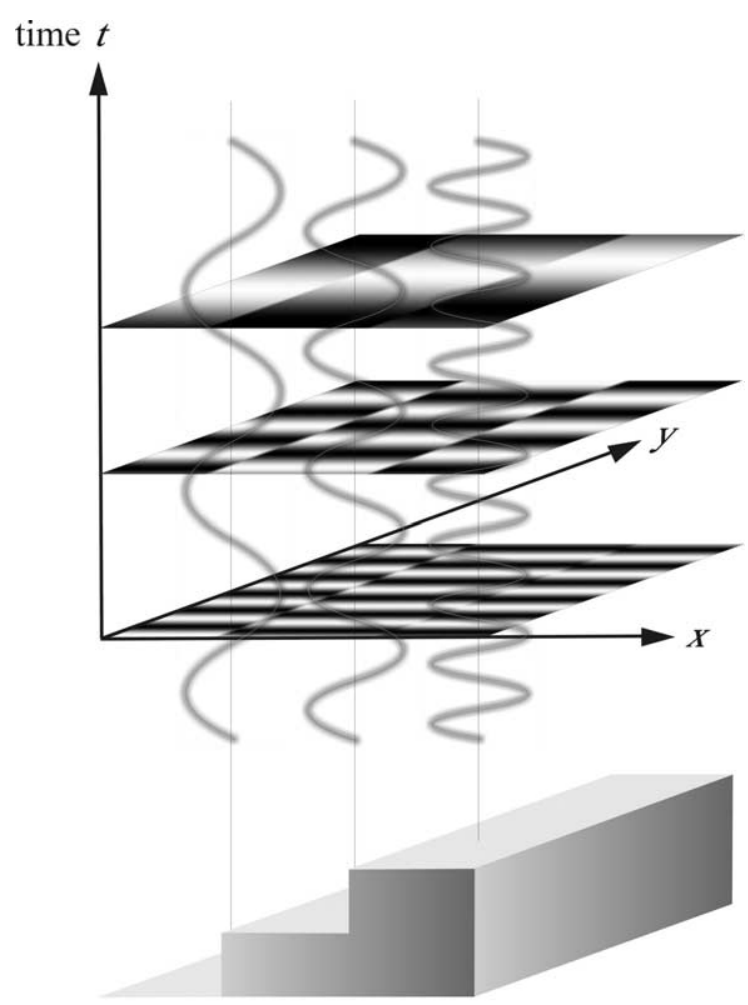

Phase function

Fig. 1. Sequence of fringe images varying the sensitivity along time. Note that the frequency of the temporal cosine signal is proportional to the phase function.

\subsection{Synchronous detection technique}

Phase detection is a task that is commonly realized in electronic communication techniques. To do so, the synchronous detection technique has been widely used. The applications of this phase detection scheme were extended to spatial phase detection of interferometric fringe patterns with spatial carrier [8-10]. The method consists in the multiplication of the signal of interest by two periodic signals in quadrature (sine and cosine waves) of the same frequency, and low-pass filtering operations. A schematic diagram of this technique is shown in Fig. 2. Applying the same principle we can determine $\beta$ from Eq. (6). For simplicity, we will describe the method in complex notation as follows. We rewrite Eq. (6) in exponential form as

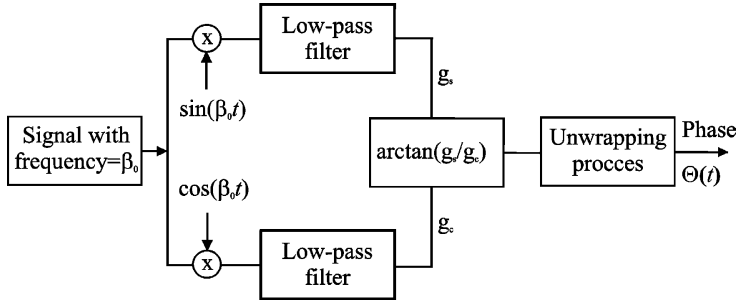

Fig. 2. Schematic diagram of the synchronous detection technique.

$$
\begin{aligned}
g(t)= & a(t)+\frac{1}{2} b(t) \exp \{\mathrm{i}[\alpha+\beta \cdot(t-1)]\} \\
& +\frac{1}{2} b(t) \exp \{-\mathrm{i}[\alpha+\beta \cdot(t-1)]\} .
\end{aligned}
$$

In this case the frequency of the periodic signal eq. (7) is $\beta$. If we multiply this equation by $\exp \left[\mathrm{i} \beta_{0}(t-1)\right]$, where the frequency $\beta_{0}$ has an arbitrary value, we will obtain

$$
\begin{aligned}
g_{e}(t)= & a(t) \exp \left[\mathrm{i} \beta_{0} \cdot(t-1)\right] \\
& +\frac{1}{2} b(t) \exp \left\{\mathrm{i}\left[\alpha+\beta \cdot(t-1)+\beta_{0} \cdot(t-1)\right]\right\} \\
& +\frac{1}{2} b(t) \exp \left\{-\mathrm{i}\left[\alpha+\beta \cdot(t-1)-\beta_{0} \cdot(t-1)\right]\right\} .
\end{aligned}
$$

Analyzing this equation we can observe that if the value of $\beta$ is sufficiently close to $\beta_{0}$ we may isolate the third term with a low-pass filtering operation and, in consequence, determine the value of $f(x, y)$. This condition can be accomplished if we establish a proper sensitivity variation which can be done controlling the experimental conditions and having a priori information about $f(x, y)$ and $k(x, y)$ (see [6]). In these conditions, we can chose $\beta_{0}=\beta_{\max }=\mu \cdot \max [f(x, y)+k(x, y)]$. Supposing that these conditions hold, we can apply a low-pass filter to the signal represented by (8), obtaining

$$
\begin{aligned}
\hat{g}_{e}(t) & =g_{e}(t) * h(t) \\
& \approx \frac{1}{2} b(t) \exp \left[-\mathrm{i}\left(\alpha+\beta \cdot(t-1)-\beta_{0} \cdot(t-1)\right)\right],
\end{aligned}
$$

where $h(t)$ represents the low-pass filter and * the convolution operation. Finally, the set of values $\Theta(t)$ can be computed as 
$\Theta(t)=\tan ^{-1}\left(-\frac{\operatorname{Im}\left\{\hat{g}_{e}(t) \exp \left[-\mathrm{i} \beta_{0} \cdot(t-1)\right]\right\}}{\operatorname{Re}\left\{\hat{g}_{e}(t) \exp \left[-\mathrm{i} \beta_{0} \cdot(t-1)\right]\right\}}\right)$.

The recovered phase $\Theta(t)$ is wrapped in the range $[-\pi, \pi]$ so that $1-\mathrm{D}$ unwrapping must be carried out. Finally $f(x, y)$ is determined from the parameter $\beta$ that is computed from the linear relationship $\Theta(t)=\alpha+\beta \cdot(t-1)$ applying linear least-squares fitting.

Applying temporal algorithms, the number of samples plays an important role in the accuracy, time processing and computer storage capacity. From the frequency view point, accuracy is incremented using a large number of samples, this is because the frequency resolution in the Fourier space is also incremented. To keep a reasonable accuracy with the lowest possible number of images, before applying this technique we have pre-processed the temporal signal with linear interpolation to 10 times the original sampling. For the convolution operation in our experiments, after testing different window filters, we found that best results are obtained using a simple average filter. It is important to appoint that apart from the least-squares fitting procedure that may be considered a filtering operation, the convolution operation implicit in this technique is beneficial for noise rejection.

\section{Numerical experiments}

In order to evaluate the performance of the method presented here, we have compared it with the Fourier method described in [6] making experiments with numerical simulations. Noise sensitivity analysis were realized making measurements on simulated fringe patterns with carrier and uniformly distributed phase-noise. We evaluate the phase with temporal series of 50 images of $50 \times 50$ pixels and considering optimal conditions of the linear sensitivity variation to apply the Fourier method as in [6] in such a way that the number of cycles of the temporal signal varied from 6 to 12. The tested function in this case was the peaks function that is included in the Matlab environment. Fig. 3 shows the normalized RMSerror by the two methods for different levels of uniformly distributed phase-noise where the range is plus/minus (+/-) the quantity indicated in the horizontal axis.

We have also compared the accuracy of both the techniques for different number of images. As in noise sensitivity analysis, we used images of $50 \times 50$ pixels and the same tested function. Fig. 4 shows the normalized RMS-error of both methods for different number of samples.

These numerical experiments show that the synchronous detection technique presents a better robustness to noise than the Fourier one due to the filtering operation implicit in this technique. Also,

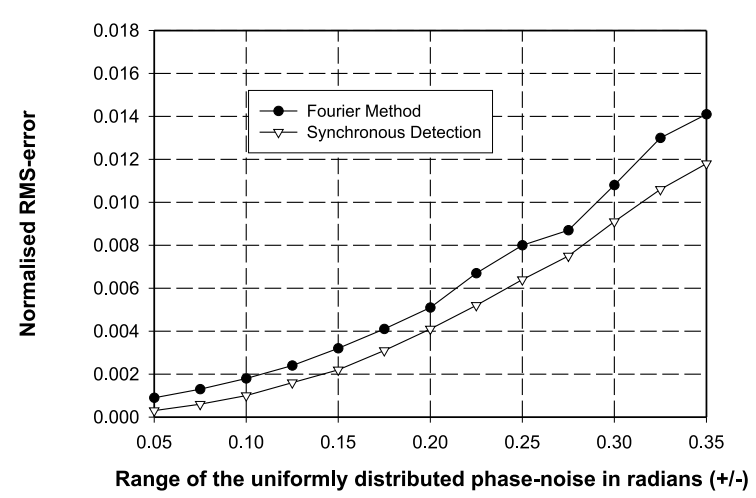

Fig. 3. Normalized RMS-error of the synchronous method applied on images of $50 \times 50$ pixels and $N=50$. Uniformly distributed phase-noise is introduced.

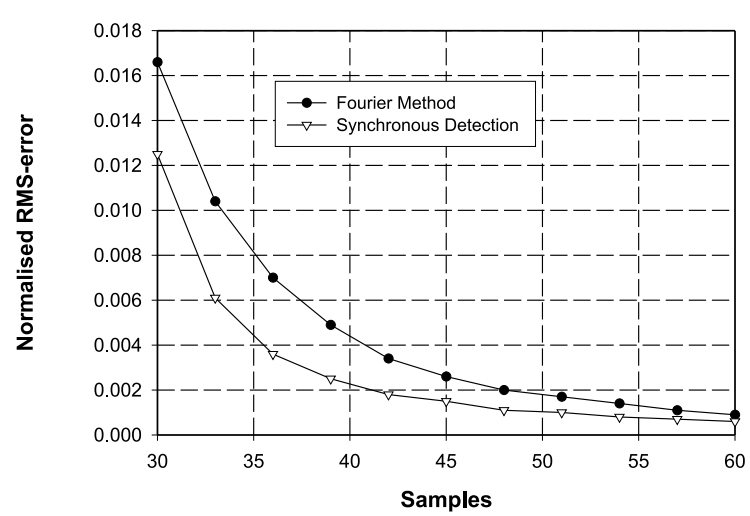

Fig. 4. Normalized RMS-error of the synchronous method applied on images of $50 \times 50$ pixels. The number of samples $N$ ranges from 30 to 60 . 
although the difference is small, the synchronous technique presents less sensitivity to low number of samples.

It is important to realize that, due to the finite size of the signal, when the filter is applied in the synchronous technique, in order to ignore phase values with large error, we just consider the phase values of the sequence $T$ to $N-T-1$, where $T$ defines the size of the filter $(2 \cdot T+1)$. In this case, the size of the window filter was selected in accordance with the discrete version of the rectangular function $h(t)=\operatorname{rect}\left(t / \beta_{0}\right)$ [10].

Finally, we have to point out one of the advantages presented by the synchronous technique compared with the Fourier method. As it is stated in [6], if we want to use the Fourier technique with a small number of frames, it is necessary to perform a preprocessing operation to the temporal signal consisting in, an interpolation and a cropping of the interpolated signal in such a way that the extension of the remaining signal corresponds to an integer number of cycles. In the case of the synchronous technique, it is only necessary to perform an interpolation operation to process the temporal irradiance signal, therefore, the computational cost is reduced and possible errors introduced by the signal cropping operation are avoided.

\section{Application and experimental results}

The temporal method proposed here has a large number of applications. For instance, we applied it to profilometry for the measurement of 3-D object shapes that is a well-known technique [4]. Fig. 5 shows the standard set-up of this application. The CCD camera captures the image of the fringes projected onto the object to be measured. In this application the phase of the fringe pattern is proportional to the height distribution and the direction of the fringes is perpendicular to the plane of the figure. The sensitivity variation is done changing the fringe frequency by means of computer generated fringes with a LCD projector.

As it is shown in [1], when sinusoidal fringes are projected over the measured object, the irradiance can be expressed as

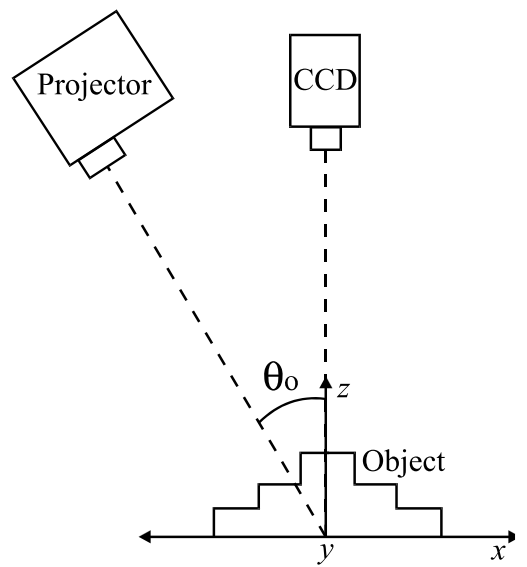

Fig. 5. Standard set-up for profilometry for the measurement of 3-D objects.

$$
\begin{aligned}
g(x, y)= & a(x, y)+b(x, y) \\
& \cdot \cos \left(\frac{2 \pi}{p}\left[z(x, y) \sin \theta_{\mathrm{o}}+x \cos \theta_{\mathrm{o}}\right]\right),
\end{aligned}
$$

where $p$ is the fringe pitch, $z(x, y)$ is the surface height and $\theta_{\mathrm{o}}$ is the projection angle formed by the optical axis of the LCD projector and the CCD camera. We must point out that Eq. (11) is true only if the height distribution of the object is negligible compared with distance from the projector to the object. According to Eq. (11), we can identify the set-up sensitivity as $\varepsilon(t)=2 \pi / p(t)$. In addition, we found that $f(x, y)=z(x, y) \sin \theta_{\mathrm{o}}$ is the quantity to be measured and $k(x, y)=x \cos \theta_{\mathrm{o}}$ is the carrier term. In these conditions, we introduce a linear sensitivity variation if the fringe pitch $p$ is changed with time in the following way:

$\frac{1}{p(t)}=\frac{1}{p_{0}}+\left(\frac{1}{p_{0}}-\frac{1}{p_{N}}\right) \cdot \frac{t}{N}$,

which leads to a linear sensitivity variation given by

$\varepsilon(t)=\frac{2 \pi}{p_{0}}+\left(\frac{1}{p_{0}}-\frac{1}{p_{N}}\right) \cdot \frac{2 \pi t}{N}$.

In our experiment, the parameters $p_{0}$ and $p_{N}$ have been determined employing the optimization procedure described in [6]. We can see in Fig. 6(a), the sinusoidal fringes projected onto the tested object, in this case a computer mouse (the values of the experimental parameters were $p_{0}=16.6 \mathrm{~mm}$, 


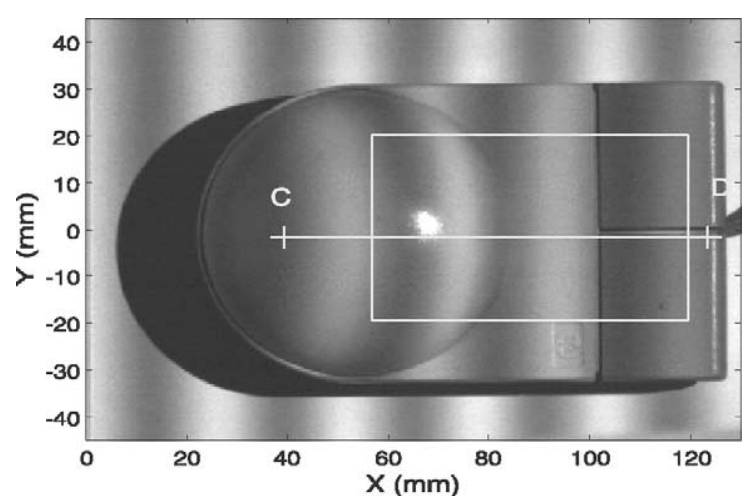

Fig. 6. Sinusoidal fringes projected over the surface of a computer mouse, used as test object.

$p_{N-1}=58 \mathrm{~mm}$ and $N=50$ frames). In Fig. 6(b) and (c), we present the results of the 3-D measurement. Fig. 6(b) shows the topography of a selected portion of the mouse surface while we have plotted in Fig. 6(c) a longitudinal height profile. From these plots, it is possible to set the accuracy in the determination of the object topography in about $2 \%$ of the maximum height of the measured surface (see Figs. 7 and 8).

We can observe experimentally that the synchronous method presents an important advantage compared with the Fourier method: We do not require to store the whole set of images because the phase $\Theta(t)$ can be computed while the fringe images are being captured which does not occur with the Fourier method.

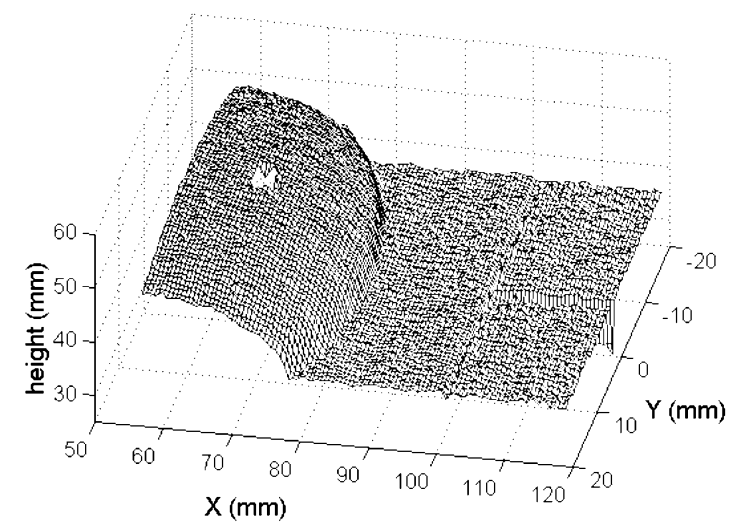

Fig. 7. 3-D perspective plot of the portion of the computer mouse surface which lies within the square (see Fig. 6).

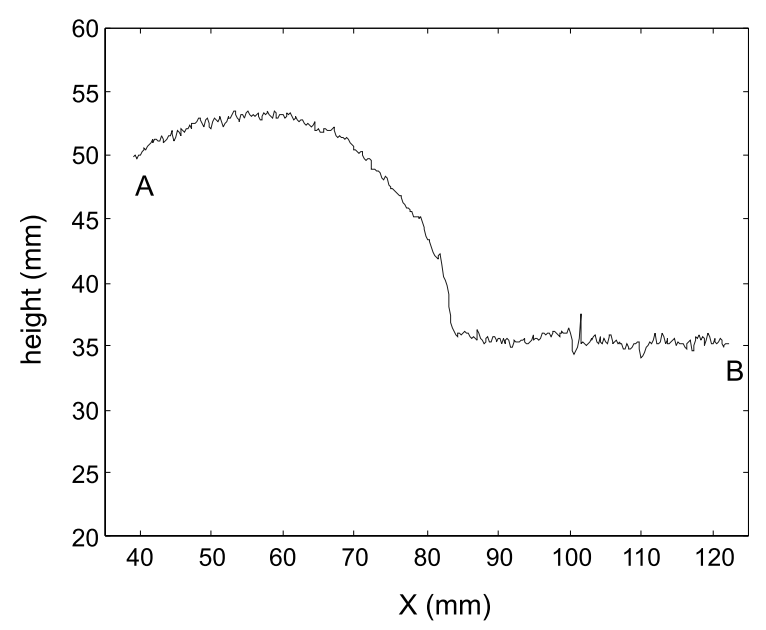

Fig. 8. A cross-section profile along the surface of the mouse (line $\mathrm{AB}$ of Fig. 6).

\section{Conclusions}

We have proposed an alternative method for the temporal analysis of fringe patterns, based on the synchronous detection technique. The main advantages that results when this technique is applied to the temporal demodulation are that: (1) It is easy to implement and no complete storage of the images is required because it is based on convolution operations. This permits a faster evaluation of the phase with a lower computational cost than Fourier techniques. (2) It presents good noise rejection characteristics due to the application of a convolution filter. (3) The presented technique is not so sensitive to the conditions of symmetry of the temporal signal as happens with the Fourier technique.

We have performed a numerical comparison between the SD and Fourier techniques. The results obtained show a better performance of the SD technique when phase noise is present. We have also applied the technique in a 3-D profilometry experiment with good results.

\section{Acknowledgements}

We acknowledge the support of the Consejo Nacional de Ciencia y Tecnologia (México) and 
the Centro de Ingenierı y Desarrollo Industrial (Querétaro, México) for the postdoctoral grant of J. Villa. We also wish to thank the economical support of the European Union, project INDUCE, BRPR-CT97-0805 and Universidad Complutense de Madrid, project PR48/01-9858.

\section{References}

[1] D.W. Robinson, G.T. Reid, Interferogram Analysis, IOP Publishing Ltd, 1993.

[2] J.M. Huntley, H. Saldner, Appl. Opt. 32 (17) (1993) 3047.
[3] J.M. Huntley, H.O. Saldner, JOSA A 14 (12) (1997) 3188.

[4] M. Takeda, H. Yamamoto, Appl. Opt. 33 (34) (1994) 7829.

[5] C. Joenathan, B. Franze, P. Haible, H.J. Tiziani, Appl. Opt. 37 (16) (1998) 3385.

[6] J.A. Quiroga, J.A. Gómez-Pedrero, J. Mod. Opt. 48 (14) (2001) 2129.

[7] H.O. Saldner, J.M. Huntley, Appl. Opt. 36 (13) (1997) 2770.

[8] Y. Ichioka, M. Inuiya, Appl. Opt. 11 (1972) 1507.

[9] K.H. Womack, Opt. Eng. 23 (4) (1984) 391.

[10] A.J. Moore, F. Mendoza, Opt. Laser Eng. 23 (1995) 319. 